\title{
Cholesterol as a Mediator of Alcohol-Induced Risks for Respiratory Disease Hospitalizations among People Living With HIV
}

\section{María José Míguez ${ }^{1 *}$, Rhonda Rosenberg ${ }^{2}$, Ximena Burbano $^{3}$ and Robert Malow ${ }^{4}$}

${ }^{1}$ Associate Professor, School of Integrated Sciences and Humanity, Florida International University, Miami, FL, USA

${ }^{2}$ Department of Health Promotion and Disease Prevention, Robert Stempel College of Public Health and Social Work and College of Medicine and School of Medicine, Florida International University, Miami, FL, USA

${ }^{3}$ School of Integrated Sciences and Humanity, Florida International University, Miami, FL, USA

${ }^{4}$ Professor, Department of Health Promotion and Disease Prevention, Robert Stempel College of Public Health and Social Work and College of Medicine and School of Medicine, Florida International University, Miami, FL, USA

\begin{abstract}
We analyzed the role of cholesterol as a potential mediator of alcohol-increased risk of respiratory infections that required hospitalization in People Living with HIV (PLWH). Using a longitudinal clinic-based design, 346 PLWH were consecutively admitted and followed at Jackson Memorial Medical Center(enrolled in the study). Following national guidelines, PLWH were stratified according to cholesterol levels: $<150 \mathrm{mg} / \mathrm{dl}$ (Hypocholesterolemia= HypoCHL), $151-200$, and $>200 \mathrm{mg} / \mathrm{dl}$ Hypercholesterolemia $=$ HyperCHL), and compared on the basis of clinical outcomes, lymphocyte phenotypes and behavioral risks. Analyses indicated that compared to HyperCHL participants, HypoCHL subjects were more likely to be hospitalized, particularly for lower respiratory tract infections (LRTI). Excessive admissions were associated with more deviant lymphocyte profiles, particularly limited NK cells. In logistic regression analyses, smoking $(\mathrm{OR}=1.5)$, HypoCHL $(\mathrm{OR}=7.7)$, and alcohol $(\mathrm{OR}=1.2)$ were predictors of $\mathrm{LRTI}$. These findings warrant further investigation of the potential use of $\mathrm{HypoCHL}$ as a risk marker, and the cost-effectiveness of switching prevention gears towards HypoCHL, alcohol and tobacco in PLWH.
\end{abstract}

Keywords: Alcohol; Cholesterol; HIV; Hospitalizations; Pneumonia; Respiratory diseases; Tobacco; Tuberculosis

\section{Introduction}

The lungs are among the most challenged organs by microbial pathogens, requiring the immune response to be both rapid and efficient [1]. As a result, lower tract respiratory infections (LRTI) are among the most common causes of morbidity, particularly among immune-compromised populations, and alcohol-abusing subjects. Despite highly active antiretroviral therapy (HAART), our team has demonstrated that LRTI continues to be a major cause of morbidity and mortality in people living with HIV [PLWH] [2-5].

Among the general population, epidemiological evidence suggests an association between alcohol use disorders (AUD) and LRTI. LRTI are caused by a broad spectrum of pathogens including virus, bacteria and mycobacteria. More than two centuries ago, Benjamin Rush, the first Surgeon General of the United States, recognized pneumonia and tuberculosis as frequent complications among subjects with AUD [6]. Since then, there have been numerous publications describing the associations between AUD and TB [7-9]. AUD deleterious effects have also been documented over other respiratory tract pathogens [10-12]. The association of AUD and LRTI were initially attributed to sociodemographic factors, nutritional deficiencies, hygienic factors, and lifestyle patterns $[13,14]$. However, subsequent studies documented that AUD exerts inhibitory effects on the adaptive immune response, particularly within the T-cell compartment [15]. Given the high cooccurrence of alcohol use disorders (AUDs) and HIV infections, our previous work focused on common immune mechanisms of HIV+ alcohol action $[16,17]$. In this regard, we have documented that AUD and HIV synergistically decreased thymic size and function, thereby resulting in abnormal $\mathrm{T}$ lymphocyte counts and function. Such alterations limit the capacity of the alcohol-consuming host to produce the needed cytokines, such as IFN- $\gamma$, IL-8, IL-17, while enhancing the levels of circulating TNF, IL-1, and IL-6 [18]. One of the central alterations related to chronic ethanol ingestion is also an impaired ability to develop specific antibodies, which markedly decreases the granulomatous response [19].

In addition to affecting the immune system, alcohol may also render the host more susceptible to TB by altering lipid homeostasis, including cholesterol depletion [20,21]. Recent studies have demonstrated that cholesteryl esters exhibit antimicrobial properties [22]. In addition, cholesterol reduction leads to other bizarre effects, such as compromised pulmonary surfactant functions [23,24]. Noteworthy, cholesterol is also a key constituent of lipid rafts, and thus essential for the correct function of immune receptors located in these regions [25]. In fact, cholesterol removal renders immune cells nonfunctional. Despite these findings, the potential role of cholesterol as mediator of the relationship between alcohol and LRTI among PLWH is still unknown.

Studying the relationship between hypocholesterolemia (HypoCHL) and acute LRTI in people with dual comorbidity could be relevant for several reasons. First, the modulatory effects of alcohol over lipids have been consistently replicated in worldwide literature and research studies. Second, HypoCHL is an early and highly prevalent phenomenon in the course of HIV disease ( $\cong 30 \%)$ [16]. Therefore, in most cases HypoCHL should be recognized as a precondition before

*Corresponding author: Maria Jose Miguez-Perez, Associate Professor, School of Integrated Sciences and Humanity, Florida International University, Miami, FL, USA, E-mail: mjmiguez@fiu.edu

Received May 15, 2011; Accepted October 18, 2011; Published October 21 2011

Citation: Míguez MJ, Rosenberg R, Burbano X, Malow R (2011) Cholesterol as a Mediator of Alcohol-Induced Risks for Respiratory Disease Hospitalizations among People Living With HIV. J AIDS Clinic Res S1:001. doi:10.4172/2155-6113.S1-001.

Copyright: (C) 2011 Míguez MJ, et al. This is an open-access article distributed under the terms of the Creative Commons Attribution License, which permits unrestricted use, distribution, and reproduction in any medium, provided the original author and source are credited. 
respiratory infections even emerged. Noteworthy, despite the initial expectations that with ART HypoCHL may disappear, this condition is still prevalent [16]. Third, in contrast to findings in the general population, attributing HypoCHL to malnutrition, or increased frailty [26-29], HIV researchers have repeatedly demonstrated, that HypoCHL is not simply a consequence of malnutrition or HIV disease progression. Fourth, hypercholesterolemia has been consistently reported as an independent predictor of morbidity and mortality. $[23,26]$ (For example, a meta-analysis of 19 cohort studies including 68,406 deaths, found an inverse correlation between total cholesterol and mortality from respiratory and gastrointestinal diseases, most of which were of an infectious origin) [30]. Fifth, it will expand therapeutic approaches of the immune system that could assist in caring for immunosuppressed hosts. Finally, no peer-reviewed studies have examined if alcohol, by altering cholesterol status, can appreciably influence immunity in PLWH and increase the risk of LRTI. Figure 1 outlines the proposed model that guided both our hypothesis and the statistical analyses, respectively.

Therefore, this study assessed cholesterol, immune status and causes of hospitalizations in individuals with single versus dual comorbidity, to increase the understanding of the precise mechanisms through which alcohol enhances the risk of being hospitalized with lower respiratory infections.

\section{Methods}

In this study, eligible participants were 1) at least 18 years old; 2) of confirmed HIV+ status; 3 ) consecutively admitted to the Miami/ Jackson Memorial Medical Center (2001-2003), a public health system with 2,482 licensed hospital and nursing home beds, and 4) were followed for at least a year in the clinics associated with Jackson Memorial Medical Center. There were multiple reasons for this inclusion criterion. First, we established this criterion in order to attain a well-defined clinical

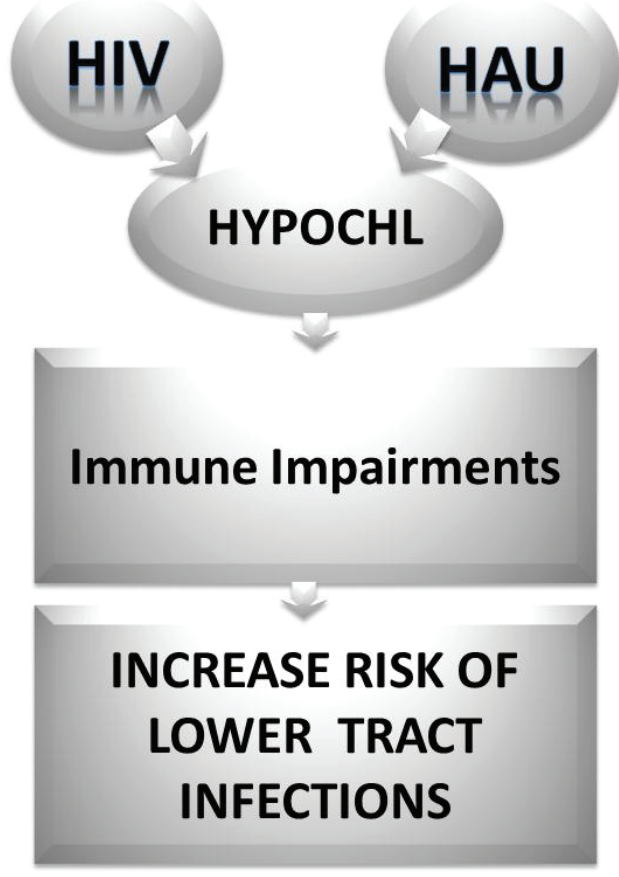

Figure 1: Proposed Model. cohort to assess the effects of hypocholesterolemia in men and women across a wide age range. Second, the restriction to those enrolled in this open-access public health system with standard treatment protocols, was to minimize social, medical and treatment inequalities that can oftentimes confound clinical outcomes. Participants were fully informed about the study, and those individuals who expressed a willingness to participate and to provide written informed consent as well as a medical release were consecutively enrolled.

Research procedures included completing structured and validated research questionnaires (e.g., sociodemographics, past and present medical history, alcohol, drugs and tobacco use), a brief medical examination (e.g., standardized procedures to measure body weight, height, and vital signs), and blood draws. Once research procedures were completed, three experienced HIV clinician/researchers abstracted participant's medical chart, and recorded the information on a standardized data collection form. Data coding included date of admission, length of stay, admission and discharged diagnosis. Routine clinical tests (i.e. pathology, laboratory, radiological procedures) performed during the hospitalization were included in the database.

\section{Exposure: lipoprotein assessment before and during the admission}

Fasting blood samples were collected at the time of hospitalization and processed within a period of 24 hours. No PLWH was acutely ill at the time of the blood draw. Total cholesterol (TC), high density lipoprotein (HDL), low density lipoprotein (LDL) and triglyceride (TG) levels were measured by routine enzymatic methods (KonePro, Konelab). Hypocholesterolemia (levels $<150 \mathrm{mg} / \mathrm{dL}$ ) was defined according to the U.S. National Cholesterol Education Program guidelines [31]. In addition, we reviewed medical records up to 12 months prior to the admission, and retrieved complete laboratory results to confirm if laboratory abnormalities were secondary to the disease process (acutely ill) or a pre-condition, thereby causing the hospitalization.

\section{Main study outcomes}

The primary endpoints of this study were causes of hospitalization, particularly respiratory infections and immune status. In order to attain a comparison with previous cholesterol studies, length of hospitalization was included as a secondary end-point.

\section{Definition of cases}

A diagnosis of a lower respiratory tract infection required at least three of the following criteria: (1) medical diagnosis, (2) X-ray findings, (3) positive culture, (4) isolation and/or visualization of the organism in sputum or bronchial wash, and, (5) response to treatment. A specific diagnosis for community-acquired pneumonia (CAP) was based on the Infectious Disease Society of America's criteria incorporating: (1) history and physical examination, (2) chest radiography, (3) sputum Gram stain and culture and, when necessary, bronchoscopy/biopsy. Pneumocystis Carinii Pneumonia (PCP) was diagnosed if at least three of the following clinical criteria were present: (1) dyspnea, (2) severe hypoxia (cyanosis or oxygen saturation $<86 \%$, as measured by pulse oximetry) and a lactate dehydrogenase level of $>1000 \mathrm{U} / \mathrm{l}$, (3) thorax X-ray compatible with PCP, (4) PCP identification or biopsy in which the pathologist suggested PCP, or, (5) response to therapy.

Following the American Thoracic Society and the Centers for Disease Control guidelines [32] the diagnosis of tuberculosis (TB) was established when two of the three following criteria were fulfilled: (1) 
acid fast bacilli (AFB) culture; (2) positive smear, and (3) presence of clinical signs/symptoms accompanied by abnormal chest X-ray or CT scan, and treatment response.

Participants were included in the non-respiratory infection category (Control Group), if the admission diagnosis did not indicate any pulmonary disease or development of respiratory illness during the hospitalization period (i.e. renal, CNS or genitourinary problems).

\section{Alcohol}

At each visit, PLWH reported alcohol intakes in the past six months using two standardized and validated brief screening questionnaires: The Alcohol Use Disorders Identification Test (AUDIT) and the Alcohol Dependence Scale (ADS). Alcohol consumption scores were computed by averaging cross products of quantity and frequency of beer/wine and hard liquor.

Hazardous drinking was defined in women who consume $\geq 7$ drinks/week (98 grams of ethanol/week) or in men who consume $\geq 14$ drinks/week (196 grams of ethanol/week).

\section{Phenotypic characterization of peripheral lymphocytes and viral status at diagnosis}

Isolated peripheral blood mononuclear cells were prepared for four-color direct immunofluorescence procedures (Becton Dickinson, San Jose, CA, USA). Flow cytometry was used to quantify the percentage and absolute numbers of T lymphocyte subpopulations CD3+/CD4+, $\mathrm{CD} 3+/ \mathrm{CD} 8+, \mathrm{B}$ lymphocytes (CD3+/CD19+) and NK cells (CD3+/ CD56+).

A reverse transcriptase polymerase chain reaction was used to quantify HIV-1 viral load (Amplicor HIV-1 Monitor, Roche Diagnostics, Indianapolis, IN, USA) in plasma from ethylenediamine tetraacetic acid (EDTA) collected blood samples, that were separated and processed within six hours of the collection period. The current version of this assay has a reportable range of $>200-750000$ RNA copies/mm of plasma. In addition, we reviewed prior immune data available in each participant's medical record, to establish their nadir CD4 cell counts and to more accurately categorize participants according to the CDC's stage of the disease classification [33].

\section{Control variables}

Each participant underwent an in-depth assessment, including a detailed interview, using standardized research questionnaires covering sociodemographic information, drug, alcohol and tobacco use habits, HIV infection-related data (i.e. stage of HIV infection), complete past and present medical, and medications history. Past medical history, and CDC's AIDS defining criteria were carefully documented [33]. A detailed medication history, including antiretrovirals and prophylaxis was obtained. Medication use history included previous exposure to antiretrovirals and lipid lowering medications. These questionnaires have been used in our previous studies [16,17]. Adherence was calculated using the standardized ACTG antiretroviral adherence questionnaire. In addition, pharmacy records were abstracted, and patient information was validated. Using standardized research questionnaires, information regarding tobacco and alcohol use (AUDIT) was obtained.

Nutritional evaluations included anthropometrics and albumin levels. Body weight and height were measured, and used to calculate body mass index (BMI = weight in kilograms divided by height in meters squared).

\section{Statistical analyses}

The data sets were analyzed using SPSS 18.0 (Statistical Package for the Social Sciences, Chicago, Illinois, USA) and $p$ values $<0.05$ were considered to be statistically significant. A gender and racially diverse population of $536 \mathrm{HIV}$-infected participants was enrolled with an overall participation rate of $97 \%$.

Descriptive statistical analyses and mean variables were compared using Student's t-tests. One-way analysis of variance (ANOVA) was employed to determine whether certain covariates (e.g., age, race/ ethnicity, education level, stress, depression) should have been included in our univariate and multivariate analyses. Correlations among the main variables of interest were examined with Pearson's coefficients. Chi-square, Student's t-test, and ANOVA, were used to evaluate differences in lipid levels between cases and controls.

Univariate analyses were used to calculate odds ratios (OR) and 95\% confidence intervals (CI). To study the relationship of cholesterol with respiratory infections, a regression model was developed. In our model, outcomes and observed covariates that were significant (all $p ' s<0.05)$ in univariate analysis were then utilized as covariates in a multivariate model along with potential confounding variables, such as gender, age, serum albumin level, smoking (yes/no), HAART (yes/no), CD4 (above or below 200 CD4 cell counts), and antibiotic prophylaxis.

\section{Results}

\section{Characteristics of the study population}

Of the 536 participants in the study, 150 of them were excluded because blood lipid values were missing. PLWH receiving cholesterollowering medication, corticoids or any cancer treatment were also excluded $(n=40)$, as medication use might have impacted the study outcomes. After these exclusions, 346 PLWH remained for the statistical analyses. The enrollees were ethnically and educationally diverse, and their overall income levels were below the national average. Also distinctive of the study population was an increase in prevalence of alcohol and tobacco use. Almost half of the study population was considered hazardous alcohol users (48\%), with a median intake of $28 \pm 4$ alcoholic drinks weekly (392 \pm 56 grams/week) for hazardous drinkers. The alcohol beverage preference was very similarly distributed; liquor was the most commonly consumed alcoholic beverage (45\%); and most liquor users $(60 \%)$ only drank liquor. One third of the participants consumed beer and $21 \%$ preferred wine. Noteworthy, HAU were twice more likely to use liquor or beer $(\mathrm{OR}=2.695 \% \mathrm{CI} 1.4-4.5, \mathrm{p}=0.0001)$ as the main alcohol beverage as compared to non-drinkers. On the other hand, non-HAU were more likely to report wine usage (OR=1.7 95\% CI 1-3.0, $\mathrm{p}=0.04$ ). Alcohol groups were very similar in sociodemographic, behavioral and clinical characteristics.

\section{Lipid profiles}

At baseline, mean (SD) values of TC (191 mg/dL \pm 9$)$, and as expected a significant proportion of the participants exhibited hypocholesterolemia (40\%) with a smaller proportion exhibiting hypercholesterolemia (9\%, see Table 1). Table 2 summarizes and displays the demographic and clinical characteristics of the sample by cholesterol groups. Groups were comparable in sociodemographic variables; however, patients with HypoCHL were more likely to be males $(\mathrm{OR}=2.1,95 \% \mathrm{CI}: 1.4-3.3, p=0.001)$. As expected, cholesterol levels were unrelated to triglyceride levels and/or to malnutrition (see Table 2). Noteworthy, participants with HypoCHL were more likely to be hazardous alcohol drinkers (OR=3.5 95\% CI 1-17, $p=0.009)$. A 


\begin{tabular}{|l|l|l|l|}
\hline Variables & Cholesterol $<150 \mathrm{mg} / \mathrm{dL}$ & Cholesterol >150 mg/dL \\
$\mathrm{N}=138$ & $\mathrm{~N}=208$ & P values \\
\hline Age in years & $41 \pm 10$ & $47 \pm 9$ & 0.8 \\
\hline Men & $90 \%$ & $56 \%$ & 0.09 \\
\hline Women & $10 \%$ & $44 \%$ & \\
\hline Black & $59 \%$ & $56 \%$ & 0.7 \\
Hispanic & $18 \%$ & $19 \%$ & \\
\hline White & $33 \%$ & $25 \%$ & \\
\hline Annual Income & & & 0.6 \\
\hline Less than $\$ 10,000$ & $89 \%$ & $88 \%$ & \\
\hline $\begin{array}{l}\text { \$11,000-\$20,000 } \\
\text { More than } \$ 20,000\end{array}$ & $9 \%$ & $10 \%$ & 0.09 \\
\hline Cigarette Smoker & 0.09 & $2 \%$ & 0.009 \\
\hline $\begin{array}{l}\text { Hazardous Alcohol } \\
\text { Use }\end{array}$ & $75 \%$ & $54 \%$ & 0.2 \\
\hline Drug Use & $42 \%$ & $45 \%$ & 0.09 \\
\hline Albumin mg/dl & $3.5 \pm 0.6$ & $39 \%$ & 0.1 \\
\hline $\begin{array}{l}\text { Body Mass Index } \\
\text { kg/m² }\end{array}$ & $26.2 \pm 6.2$ & $3.8 \pm 0.7$ & 0.3 \\
\hline HAART & $59 \%$ & $27.4 \pm 6.7$ & \\
\hline Prophylaxis & $19 \%$ & $63 \%$ & \\
\hline
\end{tabular}

Values are means \pm SD or percentages.

Table 1: Baseline Sociodemographics for PLWH with and without Hypocholesterolemia.

\begin{tabular}{|c|c|c|c|c|}
\hline Variables & $\begin{array}{c}\text { At Admission } \\
n=211\end{array}$ & $\begin{array}{c}\text { Respiratory } \\
\text { Infections } \\
n=67\end{array}$ & $\begin{array}{c}\text { Other causes of } \\
\text { Hospitalization } \\
n=144\end{array}$ & Pre-admission \\
\hline Hypocholesterolemia & $40 \%$ & $58 \%$ & $33 \%$ & $35 \%$ \\
\hline Hypercholesterolemia & $9 \%$ & $4 \%$ & $11 \%$ & $8 \%$ \\
\hline Normal Cholesterol & $51 \%$ & $38 \%$ & $56 \%$ & $67 \%$ \\
\hline Hypocholesterolemia & $40 \%$ & $58 \%$ & $33 \%$ & $35 \%$ \\
\hline Hypercholesterolemia & $9 \%$ & $4 \%$ & $11 \%$ & $8 \%$ \\
\hline Normal Cholesterol & $51 \%$ & $38 \%$ & $56 \%$ & $67 \%$ \\
\hline
\end{tabular}

Table 2: Cholesterol Status.

similar tendency was observed for HypoCHL to be smokers $(\mathrm{OR}=2.2$, 95\% CI 1-7, $p=0.09$ ).

\section{Causes of hospitalization}

Respiratory diseases accounted for approximately half (49\%) of the yearly admissions. CAP was the main cause of respiratory illnesses $(52 \%, n=251)$ and hospital admissions. Pneumocystis jiroveci continued to be an important cause of respiratory infection $(24 \%$, $n=65$ ). In $9 \%$ of the study participants, mycobacterium tuberculosis was cultured. In $3 \%$ of the study group, the discharged diagnosis was bronchitis/croup or asthma. The remaining half was hospitalized for gastrointestinal diseases (16\%), central nervous system disorders (11\%) and renal insufficiency (8\%). The other $16 \%$ was distributed among cardiovascular diseases, genitourinary conditions, and a small percentage of traumas.

\section{Lipid status}

The mean cholesterol level of the sample was $170 \pm 58 \mathrm{mg} / \mathrm{dl}$ (75-397 $\mathrm{mg} / \mathrm{dl})$, with approximately half (51\%) of the participants exhibiting normal values. Only $9 \%$ of the group exceeded $250 \mathrm{mg} / \mathrm{dl}$ of total cholesterol, and 40\% exhibited HypoCHL. As illustrated in Table 2, additional analyses indicated that prevalence of hospitalizations and LRTI were notably different by cholesterol groups, with HypoCHL showing higher risks.
To further probe if HypoCHL was only a consequence of an acute illness, or a pre-condition, surveillance (by medical chart abstraction) was extended to 180 days pre-hospitalization. Of the 346 PLWH, 211 had a pre-admission cholesterol value. As depicted in Table 1, in a sizeable subgroup, low cholesterol levels were a precondition, which was exacerbated by the acute illness. Of relevance, the illness-related average change of total cholesterol was $10.3 \pm 3 \mathrm{mg} / \mathrm{dl}$ in the HypoCHL group and $-8.4 \pm 7 \mathrm{mg} / \mathrm{dl}$ in the normal control group.

\section{Cholesterol and respiratory infections}

As illustrated in Figure 2, subjects with HypoCHL were three times more likely to be hospitalized with CAP than those with normal TC (CI: 1.1-9.9, $\mathrm{p}=0.05$ ). Additional analyses indicated that participants with LDL-C below $100 \mathrm{mg} / \mathrm{dl}$ were three times more likely to be hospitalized with CAP than control participants ( $\mathrm{OR}=3,95 \% \mathrm{CI}: 1.3-7.9, \mathrm{p}=0.01)$.

In addition, a greater risk of being admitted with $\mathrm{TB}$ was observed in those having HypoCHL ( $\mathrm{OR}=4.9$ : 95\% CI: 1.5-18.9, $\mathrm{p}=0.0008$ ). Moreover, PLWH with LDL-C below $100 \mathrm{mg} / \mathrm{dl}$ were six times more likely to be hospitalized for TB than the controls (OR=5.6 95\% CI: $1.5-22, \mathrm{p}=0.001$ ). However, hospitalization for PCP was unrelated to cholesterol levels. The clinical relevance of these findings was further explored. Although JMH possesses standardized treatment protocols, PLWH with hypocholesterolemia spent more days in the hospital than their corresponding controls $(4.4 \pm 4$ vs. $9 \pm 2.3, \mathrm{p}=0.05)$. These findings are in accordance with a previous report, indicating that cholesterolrich diet accelerates the bacteriological sterilization of sputum [34].

\section{Immune response by respiratory disease and cholesterol status}

NK cell numbers were significantly correlated with cholesterol levels $\left(\mathrm{r}^{2}=0.4, \mathrm{p}=0.009\right)$. Noteworthy, hospitalization for CAP $\left(\mathrm{r}^{2}=-0.2\right.$, $p=0.05)$ and tuberculosis $\left(r^{2}=-0.3, p=0.002\right)$ were inversely associated with cholesterol levels.

Relative to non-HypoChl, HypoCHL participants tended to have fewer CD 4 counts ( $93 \pm 88$ vs. $278 \pm 191$ cells $/ \mu L, p=0.06$ ). Nonetheless, $10 \%$ of PLWH with more than 500 CD 4 cell counts exhibited HypoCHL. Additional analyses were performed to further explore if low cholesterol values were a reflection of a more advanced stage of the disease. First, we analyzed the number of years participants lived with HIV, but no significant differences were found $(7.5 \pm 1.6$ vs. $6.2 \pm 1.5$ years). Then, the relationship between cholesterol and immune parameters was separately analyzed in those with CD4 cell counts above and below 200. Among those below 200 (i.e., with AIDS), univariate analyses indicated that the HypoCHL group was twice as

\section{Odds Ratio}

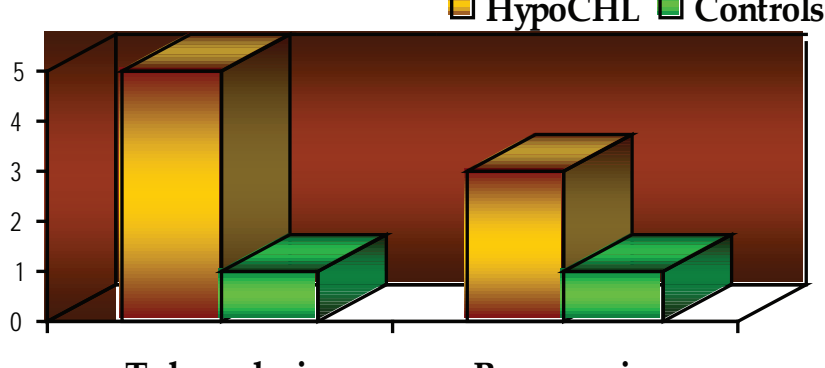

Tuberculosis

P neumonia

Figure 2: Respiratory Infections by Cholesterol Groups. 
likely to have CD4 cell counts below 50 cells than the control group (95\% CI: 1.0-4.3, $p=0.05$ ), and three times as likely to have less than 200 CD4 cell counts ( $95 \%$ CI: $1.1-3.1, p=0.01)$.

Analysis of immunological data also revealed that the percentage of NK cells was also significantly lower in Hypo CHL patients than controls ( $12.2 \pm 6$ vs. $18 \pm 11, p=0.03)$. A similar finding was evident among those with > 200 CD4 counts; for example, the mean CD4 of HypoCHL participants was $245 \pm 37$ cell counts, which contrasted with $627 \pm 381$ cell counts in those with normal cholesterol levels. NK cell percentages remained significantly different between the groups ( $17 \pm 10$ vs. $15 \pm 5, p=0.05$ ). However, percentages of CD 8 and B cells were similar between the groups.

\section{Other potential confounders}

As depicted in Table 1, there were no significant differences between the groups in antiretroviral and prophylaxis treatment before hospitalization. As illustrated in Table 3, other potential confounders, such as age, malnutrition and cigarette smoking, which may explain the observed differences, were evaluated. Interest in these associations was related to previous reports, which described an increased risk of respiratory infections associated with these same confounding factors.

\section{Multivariate analyses}

Based on clinical and statistical considerations, we selected an 8-variable model of tuberculosis, community acquired pneumonia in PLWH, including male sex, age, CD4 <200 cells, smoking, hypocholesterolemia, alcohol use and malnutrition. As shown in Table 4, HypoCHL carriers had a substantial risk of being hospitalized for lower tract respiratory infections. Multivariate regression analysis specific for tuberculosis showed that there was an inverse relation between NK numbers and risks of hospitalization. Noteworthy, for a CAP, in addition to low cholesterol, tobacco and alcohol use, individuals with altered CD8 cells showed elevated odds ratios.

\begin{tabular}{|l|l|l|l|l|l|l|l|l|l|}
\hline \multicolumn{3}{|c|}{ CAP } & \multicolumn{3}{l|}{ PCP } & \multicolumn{2}{l|}{ TB } \\
\hline MODEL & OR & $\begin{array}{l}95 \% \\
\text { Cl: }\end{array}$ & $\begin{array}{l}p \\
\text { Value }\end{array}$ & OR & $\begin{array}{l}95 \% \\
\text { Cl: }\end{array}$ & $\begin{array}{l}p \\
\text { Value }\end{array}$ & OR & $\begin{array}{l}95 \% \\
\text { Cl: }\end{array}$ & $\begin{array}{l}p \\
\text { Value }\end{array}$ \\
\hline $\begin{array}{l}\text { Malnutri- } \\
\text { tion }\end{array}$ & 1.1 & $0.6-2.1$ & 0.7 & 1.6 & $0.6-3.7$ & 0.3 & 2.9 & $1.1-7.7$ & 0.01 \\
\hline Smoking & 1.6 & $1.2-4$ & 0.02 & 2.1 & $1.1-2.2$ & 0.001 & 1.2 & $0.6-2.5$ & 0.6 \\
\hline $\begin{array}{l}\text { Hazard- } \\
\text { ous } \\
\begin{array}{l}\text { Alcohol } \\
\text { use }\end{array}\end{array}$ & 2.3 & $1.4-3.5$ & 0.001 & 1.1 & $0.8-1.6$ & 0.6 & 1 & $0.7-1.8$ & 0.7 \\
\hline Smoking & 1.3 & $0.9-1.8$ & 0.15 & 1.5 & $0.8-2.9$ & 0.2 & 1.9 & $0.8-5$ & 0.15 \\
\hline Male & 0.9 & $\begin{array}{l}0.8- \\
1.14\end{array}$ & 0.6 & 1.1 & $0.8-1.6$ & 0.5 & 1.3 & $1-1.6$ & 0.1 \\
\hline
\end{tabular}

Table 3: Univariate Analyses of Other Risk Factors Associated with Respiratory Infections.

\begin{tabular}{|l|l|l|l|}
\hline \multicolumn{4}{|l|}{ Predictors of being hospitalized with either tuberculosis or community acquired pneumonia } \\
\hline MODEL & MULTIVARIATE RR & $\mathbf{9 5 \%}$ CI: & p Value \\
\hline Hypocholesterolemia & 7.7 & $2.1-27$ & 0.002 \\
\hline Hazardous alcohol & 1.2 & $1.3-1.9$ & 0.01 \\
\hline CD4 <200 & 1.2 & $0.9-1.6$ & 0.1 \\
\hline Malnutrition & 1.1 & $0.8-1.4$ & 0.5 \\
\hline Age $>$ 50 years old & 1.15 & $0.94-1.5$ & 0.2 \\
\hline Tobacco & 1.5 & $1.2-1.8$ & 0.001 \\
\hline
\end{tabular}

Table 4: Multivariate Analyses for Being Hospitalized with Community acquired pneumonia or Tuberculosis.

\section{Discussion}

To our knowledge, the present study is the first evaluation of the clinical and immunological effects of hypocholesterolemia as a mediator of alcohol-induced clinical risks in PLWH. Of special significance, the data indicates that compared to PLWH with normal cholesterol levels, those with HypoCHL are at greatest risk of hospitalizations, particularly due to lower respiratory tract infections. These results have clinical practice implications, given that respiratory infections constitute a medical challenge during the HAART era. In addition, the HypoCHL group has an extended number of days in the hospital. These findings are highly significant given that hospital bed days are an important component of excess health care costs among this population. This information should be particularly valuable for the scientific and the public health communities in developing preventive strategies, and updated clinical guidelines.

Our data further illustrated an increased risk of hospitalizations associated with tuberculosis and community acquired pneumonia in PLWH with hypocholesterolemia. However, the risk of developing PCP does not increase, suggesting that cholesterol risks are most likely pathogen-specific. Nevertheless, some studies have not found a relationship between low cholesterol with pneumonia, influenza, respiratory diseases or with hospitalizations [35,36]. However, most studies lacking significance were solely among young individuals, while others were frequently performed exclusively in older individuals (in our specific case, it was all inclusive) $[35,36]$. Thus, discrepancies could be attributed to differences in the study population. In addition, inconsistencies could also be attributed to using different cut off points to define HypoCHL, suggesting that risks are dose-related.

Although the observational nature of our data precludes causal inferences, several facts suggested that this could be the case. First, there is a temporal relationship where medical chart review demonstrated that hypocholesterolemia predated the onset of respiratory infections and indicated that HypoCHL was a chronic condition. This is a highly relevant finding because transient HypoCHL is not necessarily relevant, and may be the consequence of a severe acute phase response, liver dysfunction, hemodilution, and even malnutrition [26,27]. Furthermore, in accordance with prior studies, our analyses revealed that HypoCHL was unrelated to malnutrition, as indicated by either normal albumin levels or body mass index. Neither was associated to more advanced stages of HIV disease.

Second, there is a biological plausibility that immune alterations are mediating our findings. Emerging evidence has indicated that immune cells and the mycobacterium tuberculosis are competing for cholesterol in order to function and survive $[34,37]$. In fact, the ability of M. tuberculosis to maintain a chronic infection seems to be linked to its ability to acquire cholesterol from the host. Additional advances in understanding the pathogenicity of $\mathrm{M}$. tuberculosis come from experimental studies demonstrating that cholesterol is necessary for macrophages to uptake and engulf mycobacteria [38]. Pieters et al. also have demonstrated that cholesterol is required for the retention of coronin-1 on mycobacteria-containing phagosomes, illustrating the importance of host cholesterol on the mycobacterium-macrophage interaction [38]. Beyond antigen presenting cells, our study provides evidence that HypoCHL affects several lymphocyte subsets in subjects infected with HIV, including NK cells. NK cells are innate immune effectors critical in the early host defense against infectious pathogens, particularly those causing TB and CAP $[39,40]$. NK are also known to be potent producers of cytokines, key to controlling bacterial load within the lungs. NK cells are an important source of interferon to 
Citation: Míguez MJ, Rosenberg R, Burbano X, Malow R (2011) Cholesterol as a Mediator of Alcohol-Induced Risks for Respiratory Disease Hospitalizations among People Living With HIV. J AIDS Clinic Res S1:001. doi:10.4172/2155-6113.S1-001.

contain a mycobacterial infection, particularly given severe immunesuppression related to advanced HIV/AIDS. As such, any impairment in the NK compartment associated with low cholesterol would likely enhance the risk of TB.

In summary, the data demonstrated that it is biologically plausible that by altering the immune system, HypoCHL may predispose PLWH to develop respiratory infectious diseases. Further supporting our postulates of a causal relationship are clinical trials, demonstrating that a cholesterol-rich diet (750 mg daily) accelerated the bacteriological sterilization of sputum when added to the four-drug antitubercular treatment.

Finally, the robust associations between HypoCHL and respiratory infections and its persistent relationship after controlling for confounders, supports the hypothesis that hypocholesterolemia is a mediator of alcohol deleterious health effects. These results also highlight the need to better understand the specific key molecular mechanisms involved in these processes, so that new targeted therapies may be developed to prevent these morbidities.

Nevertheless, it is important to recognize some limitations of the study design. First, the results are limited to the study population. Second, the observational nature of our data precludes causal inferences. However, considering that a sizable proportion of the PLWH develops HypoCHL, it would be valuable to assess in clinical trials if cholesterol screening and preventive interventions, could in fact, ameliorate or decrease the risks. At the very least, our article should raise awareness of HypoCHL's possible deleterious effects on PLWH, particularly in light of the increasing use of lipid lowering medications, and thus stimulated further research in this area.

\section{Acknowledgement}

This study was funded in part by NIH NIAAA 1R01AA018095-01 and NIH NIAAA 3R01AA017405-0251 MJM.

\section{References}

1. Forbes LR, Haczku A (2010) SP-D and regulation of the pulmonary innate immune system in allergic airway changes. Clin Exp Allergy 40: 547-562.

2. Miguez-Burbano MJ, Burbano X, Ashkin D, Pitchenik A, Allan R, et al. (2003) Impact of tobacco use on the development of opportunistic respiratory infections in HIV seropositive patients on antiretroviral therapy. Addiction Biology 8: 39-43.

3. Crum-Cianflone NF, Grandits G, Echols S, Ganesan A, Landrum M, et al. (2010) Trends and Causes of Hospitalizations among HIV-infected Persons during the Late HAART Era: What is the Impact of CD4 Counts and HAART Use? J Acquir Immune Defic Syndr 54: 248-257.

4. Dufour V, Cadranel J, Wislez M, Lavole A, Bergot E, et al. (2004) Changes in the pattern of respiratory diseases necessitating hospitalization of HIV-infected patients since the advent of highly active antiretroviral therapy. Lung 182: 331-341.

5. Berry SA, Gebo KA, Moore RD, Manabe YC (2010) A high risk of hospitalization immediately follows HAART initiation. HIV Medicine Volume 11, Issue 5, pages 289-298.

6. Rush B (1785) An inquiry into the effects of ardent spirits upon the human body and mind: With an account of the means of preventing, and of the remedies for curing them. $8^{\text {th }}$ edition.

7. World Health Organization (2007) Tuberculosis facts. Accessed January 14, 2008.

8. Jacobson JM (1992) Alcoholism and tuberculosis. Alcohol Health Res World 16: 39-45.

9. Szabo G (1997) Alcohol and susceptibility to tuberculosis. Alcohol Health Res World 21: 39-41.

10. Gamble L, Mason CM, Nelson S (2006) The effects of alcohol on immunity and bacterial infection in the lung. Med Mal Infect. 36: 72-77.
11. Zhang P, Bagby GJ, Happel KI, Summer WR, Nelson S (2002) Pulmonary host defenses and alcohol. Front Biosci 7: d1314-1330.

12. Skerrett SJ, Niederman MS, Fein AM (1989) Respiratory infections and acute lung injury in systemic illness. Clin Chest Med 10: 469-502.

13. Kang DR, Kim YK, Park MS, Kim YS, Ko DH, et al. (2008) Low levels of serum cholesterol and albumin and the risk of community-acquired pneumonia in young soldiers. Int J Tuberc Lung Dis 12: 26-32.

14. Kalantar-Zadeh K, Ikizler TA, Block G, Avram MM, Kopple JD (2003) Malnutrition inflammation complex syndrome in dialysis patients: causes and consequences. Am J Kidney Dis 42: 864-881.

15. Meyerholz DK, Edsen-Moore M, McGill J, Coleman RA, Cook RT, et al. (2008) Chronic alcohol consumption increases the severity of murine influenza virus infections. J Immunol 181: 641-648.

16. Míguez Burbano MJ, Lewis JE, Malow R (2009) Alcohol and race/ethnicity elicit different changes in lipid profiles in HIV-infected individuals receiving highly active antiretroviral therapy. J Assoc Nurses AIDS Care 20: 176-183.

17. Míguez-Burbano MJ, Lewis JE, Fishman J, Asthana D, Malow RM (2009) The influence of different types of alcoholic beverages on disrupting highly active antiretroviral treatment (HAART) outcome. Alcohol Alcohol 44: 366-371.

18. Deviere J, Content J, Denys C, Vandenbussche P, Schandene L, et al. (1989) High interleukin-6 serum levels and increased production by leucocytes in alcoholic liver cirrhosis. Correlation with IgA serum levels and lymphokines production. Clin Exp Immunol 77: 221-225.

19. Happel KI, Nelson S (2005) Alcohol, immunosuppression, and the lung. Proc Am Thorac Soc 2: 428-432.

20. Marcén R, Teruel JL, de la Cal MA, Gámez C (1997) The impact of malnutrition in morbidity and mortality in stable haemodialysis patients. Nephrol Dial Transplant 12: 2324-2331.

21. Memon RA, Staprans I, Noor M, Holleran WM, Uchida Y, et al. (2000) Infection and inflammation induce LDL oxidation in vivo. Arterioscler Thromb Vasc Biol 20: $1536-1542$.

22. Lee JT, Jansen M, Yilma AN, Nguyen A, Desharnais R, et al. (2010)Antimicrobia lipids: novel innate defense molecules are elevated in sinus secretions of patients with chronic rhinosinusitis. Am J Rhinol Allergy 24: 99-104.

23. Rooney SA (1985) The surfactant system and lung phospholipid biochemistry Am Rev Respir Dis 131: 439-460.

24. Wilsher ML, Hughes DA, Haslam PL (1988) Immunoregulatory properties of pulmonary surfactant: influence of variations in the phospholipid profile. Clinical \& Experimental Immunology 73: 117-122.

25. Dykstra M, Cherukuri A, Sohn HW, Tzeng SJ, Pierce SK (2003) Location is everything: Lipid Rafts and Immune Cell Signaling. Annu Rev Immunol 21: 457-481.

26. Ives DG, Bonino P, Traven ND, Kuller LH (1993) Morbidity and Mortality in Rura Community-Dwelling Elderly With Low Total Serum Cholesterol. J Gerontol 48 : M103 -107.

27. Bonnefoy M, Abidi H, Jauffret M, Garcia I, Surrace JP, et al. (2002) Hypocholesterolemia in hospitalized elderly: relations with inflammatory and nutritional status. Rev Med Interne 23: 991-998.

28. Ceppa F, Merens A, Burnat P, Mayaudon H, Bauduceau B (2008) Military community: a privileged site for clinical research: Epidemiological Study of Metabolic Syndrome Risk Factors in the Military Environment. Mil Med 173 960-967.

29. Gordon BR, Parker TS, Levine DM, Saal SD, Wang JC, et al. (2001) Relationship of hypolipidemia to cytokine concentrations and outcomes in critically ill surgical patients. Crit Care Med 29: 1563-1568.

30. Ravnskov U (2002) A hypothesis out-of-date: the diet-heart idea. J Clin Epidemiol 5: 1057-1063.

31. US Department of Health and Human Services (2002) U.S. National Cholestero Education Program guidelines.

32. Diagnostic Standards and Classification of Tuberculosis in Adults and Children This official statement of the American Thoracic Society and the Centers for Disease Control and Prevention was adopted by the ATS Board of Directors, July 1999. This statement was endorsed by the Council of the Infectious Disease Society of America, September 1999. (2000) Am J Respir Crit Care Med 161: 1376-1395 
Citation: Míguez MJ, Rosenberg R, Burbano X, Malow R (2011) Cholesterol as a Mediator of Alcohol-Induced Risks for Respiratory Disease Hospitalizations among People Living With HIV. J AIDS Clinic Res S1:001. doi:10.4172/2155-6113.S1-001.

Page 7 of 7

33. Centers for Disease Control and Prevention (1992) 1993 revised classification system for HIV infection and expanded surveillance case definition for AIDS among adolescents and adults. MMWR Recomm Rep 41: 1-19.

34. Pérez-Guzmán C, Vargas MH (2006) Hypocholesterolemia: a major risk factor for developing pulmonary tuberculosis? Med Hypotheses 66: 1227-1230.

35. Iribarren C, Jacobs DR Jr, Sidney S, Claxton AJ, Gross MD, et al. (1997) Serum total cholesterol and risk of hospitalization, and death from respiratory disease. Int J Epidemiol 26: 1191-1202.

36. Iribarren C, Jacobs DR Jr, Sidney S, Claxton AJ, Feingold KR (1998) Cohort study of serum total cholesterol and in-hospital incidence of infectious diseases. Epidemiol Infect 121: 335-347.
37. Pandey AK, Sassetti CM (2008) Mycobacterial persistence requires the utilization of host cholesterol. Proc Natl Acad Sci U S A 105: 4376-4380.

38. Gatfield J, Pieters J (2000) Essential role for cholesterol in entry of mycobacteria into macrophages. Science 288: 1647-1650.

39. Bozzano F, Costa P, Passalacqua G, Dodi F, Ravera S, et al. (2009) Functionally relevant decreases in activatory receptor expression on NK cells are associated with pulmonary tuberculosis in vivo and persist after successful treatment. International Immunology 21: 779-791.

40. Krensky AM (2000) Granulysin: a novel antimicrobial peptide of cytolytic T lymphocytes and natural killer cells. Biochem Pharmacol 59: 317-320. 